\title{
Performance Evaluation of Different Encryption Schemes on Portable and Mobile Platforms
}

\author{
Fadi Al-Masalha*, Rogelio Hasimoto $\$$, and Ashfaq Khokhar" \\ Departments of $\mathrm{CS}^{*}$ and $\mathrm{ECE}^{\mathbb{}}$ \\ ${ }^{\$}$ Center for Research in Mathematics (CIMAT), \\ University of Illinois at Chicago \\ Chicago, USA \\ (falmas2,ashfaq)@uic.edu \\ Jalisco s/n, Col. Mineral de Valenciana, Guanajuato, \\ Gto, México 36240. \\ hasimoto@cimat.mx
}

\begin{abstract}
Encryption is becoming an increasingly dominant component in online and mobile applications. While robust security is an important property desired in encryption schemes, it is equally important for such schemes to be highly energy efficient. In this paper we evaluate traditional AES implementations and non-traditional Chaotic Maps based encryption schemes. Our experiments reveal that Chaotic Maps based schemes outperform the traditional AES implementation in terms of CPU usage, encryption speed, and energy consumption. Particularly they consume 300-400 \% less CPU, and have over $250 \%$ faster encryption speed. In addition, the energy usage of the Chaotic scheme for a given input rate or size is 3 times better compared to the other systems considered. However, the performance also depends on the floating point capability of the platform; a suitable scheme may be chosen depending on the battery and CPU power of platform. The performance results reported in this paper are based on experiments on desktops, laptops, netbooks, and PDAs.
\end{abstract}

Keywords-encryption; AES; Chaoic Maps;mobile devices;

\section{INTRODUCTION}

Handheld devices using rechargeable battery units represent one of the most convenient ways to access user private information (bank accounts, bill paying, online browsing, video streaming, etc.) on anytime-anywhere basis. These advantages come with intrinsic pollution and security risks. From the pollution perspective, battery should provide longer life, and from the security perspective wireless, since data transfer is ubiquitous, it may be intercepted by malicious intruders. Protecting private user information has been one of the major concerns during the last decade, and more recently the efforts are being concentrated on mobile devices. This problem is further exacerbated by the growing prevalence of multimedia applications, where, at times, secure streaming of large video/audio streams is desired. There are two particular issues in securing information on mobile devices: 1) lack of processor power to manage complex encryption schemes, particularly for live streaming, and 2) limited battery energy to manage not only the encryption process, but also energy consuming computations such as video/audio encoding/decoding. The problem gets worse in a more constrained environment such as sensor networks [1], where memory storage is limited and battery energy is difficult to replenish. Therefore, it is imperative that the encryption process must be simple (without overlooking security) and energy efficient to satisfy current mobility demands while promoting green computing practices.

Energy consumption of traditional encryption schemes such as DES, RC, IDEA, GOST, and (different implementations of) AES has been analyzed in [2-5]. The energy consumption of for DES, GOST, and IDEA was evaluated in [2], and found to be proportional to the number of rounds used in the encryption process. From these models, GOST obtained the smallest slope, and IDEA the maximum slope. An interesting result in [3] is that, the Two Fish algorithm obtained the best rating for the encryption, RC6 for decryption, and Serpent the fastest execution time. Traditional encryption schemes have also been evaluated on a joint effort between security and wireless network throughput in order to decrease the overall energy consumption during transmission [4]. Recently, nontraditional encryptions schemes, particularly those based on Chaotic Maps [5] have been proposed. While such schemes --shown to be equally robust in terms of security properties-- have not been formally tested and compared against the traditional ones. In this paper, we present a comprehensive evaluation of different encryption schemes in terms of speed, CPU usage, and energy consumption, on multiple computing platforms (desktops, laptops, netbooks, and PDAs), and provide a guidelines to their usability under different scenarios.

Chaotic encryption, originally proposed for analog communications (early 90s) has evolved as a viable alternative to traditional encryption (AES, DES, etc.) for secure digital communication of text, images and more recently voice and video applications. It consists of a digital generator of chaos (nonlinear dynamic function), whose output transforms input data (plaintext) into an independent masked output (ciphertext) with uniform distribution. Digital Chaotic Systems (DCS) have many of the good 
properties required in cryptography; the most prominent are sensitivity to parameters, sensitivity to initial conditions and unpredictable trajectories [6]. The first two properties are related to diffusion, and the last one to confusion in the cryptographic nomenclature. Confusion is intended to make the relationship between ciphertext and plaintext statistically independent, while diffusion is intended to spread out the influence of a single plaintext digit over many ciphertext digits to hide the statistical structure of the plaintext. One of the main concerns of chaotic encryption is the use floatingpoint arithmetic, which may require additional hardware (and compatible arithmetic operations between different processors) in order to run efficiently. Regardless of this, it is foreseen as a viable alternative to traditional encryption schemes, especially for real-time multimedia applications.

The main goal of this work is to evaluate the performance and energy consumption of popular AES implementations [7-9] and the nontraditional chaotic encryption scheme proposed in [5] on mobile devices (notebook, netbook, and PDAs). The analysis is performed on a diverse set of platforms and experimental results are reported for multiple encryption schemes under different usage scenarios.

\section{ENCRYPTION SCHEMES}

For the sake of completeness, in this section we provide a brief overview of AES and the chaotic encryption scheme proposed in [5]. In the case of AES, our main concentration is on the latest implementation proposed by Bernstein at al. [9], but we also include other popular implementations of AES by Gladman [8], and PolarSSL [10].

\section{A. Advanced Encryption Standard (AES) (Heading 2)}

AES is the encryption standard adopted by the U.S. government in 2001, for the encryption of electronic data. It is based on based on the original proposal of Rijndael [10] with fixed block length support AES-128, AES-192, and AES-256. Following the description in [11], AES operates on an internal state of 128 bits, which is initially set to the plaintext block, and after transformations, becomes the output ciphertext block. The state is organized in a $4 \times 4$ array of 8-bit bytes, which is transformed according to a round function $\mathrm{N}_{r}$ times. The number of rounds is $\mathrm{N}_{r}=10$ for 128bit keys, $\mathrm{N}_{r}=12$ for 192-bit keys, and $\mathrm{N}_{r}=14$ for 256-bit keys. In order to encrypt, the state is first initialized, then the first 128-bits of the key are xored into the state, after which the state is modified $\mathrm{Nr}_{\mathrm{r}}-1$ times according to the round function, followed by the slightly different final round.

The round function consists of four steps: SubBytes, ShiftRows, MixColumns and AddRoundKey (except for the final round which omits the MixColumns step). Each step operates on the state, at each round $r$, as follows:

1. SubBytes: substitutes every entry (byte) of the state with an S-box entry,

2. ShiftRows: cyclically left shifts every row $i$ of the state matrix by i; $0 \leq i \leq 3$,
3. MixColumns: multiplies each column, taken as a polynomial of degree less than 4 with coefficients in $F_{28}$, by a fixed polynomial modulo $\mathrm{x}^{4}+1$,

4. AddRoundKey: XORs the r-th round key into the state.

Each transformation has an inverse from which decryption follows in a straightforward way by reversing the steps in each round: AddRoundKey (inverse of itself), InvMixColumns, InvShiftRows, and InvSubBytes.

The key expansion into the $\mathrm{Nr}$ 128-bit round keys is accomplished using a key scheduling algorithm, the details of which can be found in [10]. The design of the key schedule allows for the full expansion to precede the round transformations, which is advantageous if multiple blocks are encrypted using the same key, while also providing the option for on-the-y key generation. On-the-fly key generation proves useful in memory constrained environments such as microcontrollers.

For 32-bit (and greater word length) processors, in [11] Daemen and Rijmen detail a fast implementation method that combines the SubBytes, ShiftRows, and MixColumns transformations into four 256-entry (each entry is 4 bytes) lookup tables, $\mathrm{T}_{\mathrm{i}}, 0 \leq \mathrm{i} \leq 3$. Following [11], the "T-table" approach reduces the round transformations to updating the j-th column according to:

$$
\left[s_{0, j}^{\prime}, s_{1, j}^{\prime}, s_{2, j}^{\prime}, s_{3, j}^{\prime}\right]^{T}=\oplus_{i=0}^{3} T_{i}\left[s_{i, j}+C_{i}\right], \text { for } 0 \leq j \leq 3,
$$

Where $s_{j, k}$ is the byte in the j-th row and k-th column of the state, and $\mathrm{C}_{\mathrm{i}}$ is a constant equivalently doing the ShiftRows in-place. After the columns are updated, the remaining transformation is AddRoundKey (which is a 4-byte look-up and xor per column). Note, however, since the $T_{i}$ 's are simply rotations of eache other, some implementations of (1) benefit from using a single table and performing the necessary rotations.

In [9], authors describe a new AES implementation that takes advantage of the architecture-dependent reduction of instructions used to compute AES and the microarchitecture-dependent reduction of cycles used for those instructions. Authors report new software speed records than previous AES implementations for different CPUs.

\section{B. Chaotic Encryption}

This section provides a broad overview of the nontraditional encryption scheme that will be used in our performance and energy consumptions tests. For more detailed explanation of the scheme see [5].

Broadly speaking, the scheme in [5] is a generalized symmetric cryptosystem based on $N$ logistic maps periodically perturbed with a three-level perturbation scheme. The first and second level perturbations make the system extremely sensitive to changes in the plaintext data, while the third level perturbation continuously renews the 
system-key. To further increase security, the system creates interdependencies between maps and ciphertexts by adding global and local feedback during the encryption process, so that new ciphertext depends on the history of previous ciphertext values in the current iteration (global feedback) and the history of previous ciphertext values within the same map (local feedback). A general diagram of the systems is presented in Fig.1.

The system takes the initial system-key of size B bits $(\mathrm{B} \geq 128$, and multiple of 32 ) to generate the $\mathrm{N}$ parameters $(\lambda)$ and initial variables $\left(\mathrm{X}_{0}\right)$ required by the logistic map $\left(X_{n}=\lambda X_{n-1}\left(1-X_{n-1}\right)\right)$. It iterates a random number of times the $\mathrm{N}$ maps using with coupling function to make the system sensitive to key changes; the final output variables are taken as the initial point to start the encryption process (this is the only step where maps are dependently iterated). Once the $\mathrm{N}$ maps have been defined, only a small window (W) of $\mathrm{m} \leq \mathrm{N}$ maps are used for encryption; after some random time the $\mathrm{W}$ moves around the $\mathrm{N}$ maps (one map or step at a time) in a circular list fashion. This process keeps the system in a nonstationary state if one or some maps get trapped in a chaotic cycle. The plaintext to ciphertext transformation is represented by the following equation:

$$
\begin{aligned}
C_{l}=\mathrm{C}_{\mathrm{W}(\mathrm{i}), \mathrm{j}}= & \left(\left(\left[P_{l}+X_{W(i), j}^{\prime}\right] \bmod 2^{n}\right) \oplus X_{W(i), j}^{\prime}\right) \\
& \oplus\left(\left[X_{W(i+i), j}^{\prime}+X_{W(i+2), j}^{\prime}\right] \bmod 2^{n}\right) \\
& \oplus\left(\left[\mathrm{C}_{\mathrm{W}(\mathrm{i}-1), \mathrm{j}}+\mathrm{C}_{\mathrm{W}(\mathrm{i}), \mathrm{j}-1}\right] \bmod 2^{n}\right),
\end{aligned}
$$

Where $P_{l}$ is the $l$ th plaintext input, $X_{W(i), j}^{\prime}$ is the corresponding integer representation of the chaotic output $X_{W(i), j}$ using $n$ bits, $C_{W(i-1), j}$ is the previous ciphertext output $(i-1)$ in current iteration ( $j$-th), and $C_{W(i), j-1}$ is the previous ciphertext output of the same $i$-th map, but from the $j^{-1}$ iteration. $C_{W(i-1), j-1}$ and $C_{W(i), j-1}$ represent the global and local feedback, respectively.

The initial global feedback $C_{W(i-1), j}$ for the $j$-th iteration takes in the last ciphertext output of the previous iteration $C_{W(m), j-1}$ to spread the system changes on to future ciphertexts and current $m$ logistic map variables. A total of $32 m$ bits are encrypted per iteration state $j$ (32 encrypted bits per map). $W_{m, k}$ is periodically rotated one map at a time by setting $k=k+1$; when $(N-k+1)<m$, the cipher index wraps around taking the corresponding first, second, up to the $m$ $(N-k+1)$ initial maps.

The corresponding decryption system can be written as:

$$
\begin{gathered}
P_{l}=\left[\left(C_{l} \oplus X_{W(i), j}^{\prime} \oplus\left[\mathrm{C}_{\mathrm{W}(\mathrm{i}-1), \mathrm{j}}+\mathrm{C}_{\mathrm{W}(\mathrm{i}), \mathrm{j}-1}\right] \bmod 2^{n}\right)\right. \\
\oplus\left(\left[X_{W(i+i), j}^{\prime}+X_{W(i+2), j}^{\prime}\right] \bmod 2^{n}\right) \\
\left.-X_{W(i), j}^{\prime}\right] \bmod 2^{n}
\end{gathered}
$$

The original scheme describes three levels of perturbations, but for the purpose of the experiment we only make use of the first perturbation level defined as:

$$
X P_{W(i), j-1}=X_{W(i), j-1}+\frac{\sum_{l=1}^{4} C_{W(m), j-1}(l)}{10^{h_{8}}}
$$

where $C_{W(m), j-1}$ is the $l$-th byte of the global feedback at the state $j-1$, and $h 8$ is the number of digits in the largest decimal number represented by 8 bits.

In the experiment set up we use a key size of $B=384$ bits $\mathrm{N}=12$ maps, $\mathrm{W}=4$, and a perturbation period of 1500 iterations. Since only 4 maps are being evaluated at a time, the ciphertext output size is 128 bits.

\section{METHODOLOGY}

To evaluate the performance of different encryption schemes reviewed in the previous section, we have developed implementations of these schemes on the following computing platforms: Dell desktop, Lenovo laptop, Asus netbook, and Nokia N800. Table 1 shows specifications of each platform. These platforms are chosen to reflect diversity in computation energy, battery capacity, and mobility characteristics. We evaluate and compare each scheme in terms of CPU usage, energy consumption, and encryption speed.

Table 1: Specifications of Different Platforms used in Experiments

\begin{tabular}{|c|c|c|c|c|}
\hline Testbed & $\begin{array}{c}\text { CPU } \\
\text { Type }\end{array}$ & $\begin{array}{c}\text { Clock } \\
\text { Speed }\end{array}$ & Memory & $\begin{array}{c}\text { Operating } \\
\text { System }\end{array}$ \\
\hline \hline Desktop & $\begin{array}{c}\text { Intel } \\
\text { Duo } \\
\text { Core } 2\end{array}$ & $2.2 \mathrm{Ghz}$ & $3 \mathrm{~GB}$ & Ubuntu8.3 \\
\hline Laptop & $\begin{array}{c}\text { Intel } \\
\text { Duo } \\
\text { Core } 2\end{array}$ & $2.2 \mathrm{Ghz}$ & $2 \mathrm{~GB}$ & Ubuntu9.1 \\
\hline NetBook & $\begin{array}{c}\text { Intel } \\
\text { Atom }\end{array}$ & $1.6 \mathrm{Ghz}$ & $1 \mathrm{~Gb}$ & $\begin{array}{c}\text { Ubuntu } \\
\text { Netbook }\end{array}$ \\
\hline $\begin{array}{c}\text { Nokia } \\
\text { N800 }\end{array}$ & $\begin{array}{c}\mathrm{TI} \\
\text { Omap } \\
2420\end{array}$ & $\begin{array}{c}333 \\
\mathrm{Mhz}\end{array}$ & $128 \mathrm{MB}$ & Maemo \\
\hline
\end{tabular}

We installed Ubuntu Linux distribution on all devices except Nokia N800 which runs Maemo[12] distribution. Maemo is a mobile operating system for Nokia PDAs based on Debian GNU/Linux operating system. Ubuntu implements battery management using the Advanced Configuration and Power Interface (ACPI), which exports battery data via the /proc/acpi/battery file system. The data values exported by ACPI expressed by millivolts and milliamps which can be converted to Watt: Given the current voltage and amps of battery using ACPI values, we compute energy consumed (in Watt Hour).

We conducted energy consumption tests on two devices only, i.e., laptop and netbook, as these are equipped with batteries, while we conducted encryption speed and CPU 
usage on all the four devices. We could not perform energy consumption tests on Nokia devices due to unavailability of power monitoring tools. Table 2 shows battery specification of laptop and netbook used in energy drain tests.

Table 2: Features of Rechargeable Batteries used in Experiments

\begin{tabular}{|l|l|l|}
\cline { 2 - 3 } \multicolumn{1}{c|}{} & Laptop & Netbook \\
\hline Battery Capacity & $4752 \mathrm{mAh}$ & $4400 \mathrm{mAh}$ \\
\hline Voltage & $11100 \mathrm{mV}$ & $11100 \mathrm{mV}$ \\
\hline Type & Lion & Lion \\
\hline
\end{tabular}

We adopted the following methodology to measure energy consumption. Each device is first fully charged and each encryption schemes is modified so that it runs in an infinite loop. For each scheme, we periodically polled (every 60 seconds) the Linux ACPI values and computed energy usage. To measure the actual energy used by encryption operation, each device is first fully charged and then left on idle running. We periodically polled (every 60 seconds) the Linux ACPI values and computed the energy consumption. The difference of the idle energy and energy consumed during encryption operation is reported as the energy consumed by the encryption scheme.

\section{RESULTS}

For the sake of clarity, speed and CPU usage on each platform are analyzed first, followed by battery dissipation and CPU usage. Fig.2a compares the CPU usage at a constant rate for different streaming rates; this means that all implementations are processing the same amount of data over the same period of time. The Chaotic encryption is $\sim 4$ times $(400 \%)$ better in CPU usage than Bernstein's and Gladman's implementations for the netbook and laptop platforms; on the desktop Bernstein's improved its performance, but still $\sim 2(200 \%)$ worse than chaotic encryption. OpenSSL performed was poor in all the tests. On the Nokia platform, Gladman's obtained the best performance. As shown in Fig.2b, the Chaotic encryption is by far the fastest scheme on mid-level systems (desktop, laptop and netbook), with an average of 2.6 (260\%) times better performance than the fastest AES implementation as of 2008 [9] (Bernstein). Among AES implementation, Bernstein's implementation obtained the best performance, except for the netbook where Gladman's did a little better. In the Nokia device, Gladman's was the best, closely followed by Bernstein's and Rogelio's chaotic scheme. We were expecting worse numbers for chaotic encryption since the core of the process consist of floating-point computation, and Nokia 800 lacks of math coprocessor in its TI Omap2420 CPU.

Fig. 3 shows the battery energy consumption on battery operated devices. Fig.3a shows the performance of encryption schemes in terms of energy usage and the amount of data encrypted. A linear behavior for all implementation is observed with AES implementation having the greatest slopes or energy consumption. For example, Bernstein's implementation encrypts 280 GBytes of data and consumes over 32 Watt Hour of battery energy. On the other hand, Chaotic scheme encrypts 800 GBytes of data and consume in the same 32 Watt Hour of the energy. Fig. 3b shows energy consumed for different data sizes. Overall, Chaotic encryption can process $300 \%-400 \%$ more information with the same energy consumption than the AES implementations. While the trend is similar across platforms, the differences are much sharper in the case of netbook. We attributed this fact to the energy characteristics of the Atom processor that is more optimized for the energy. These results show that choice of the encryption scheme for a desired rate should depend on the hardware and battery capabilities of the platform.

\section{CONCLUSION}

In this paper we have evaluated the performance of multiple encryption schemes in terms of CPU usage, battery energy usage, and encryption speed. Our results shows Chaotic Maps based scheme are significantly superior in terms of speed and energy encryption. However, they yield poor performance when the target platform does not have math co-processor. Similarly, platforms such as netbook that are tuned for mobile and battery operated environments give superior performance for optimized codes. In our future work, we plan to apply these techniques for real time encryption of live video streams that also exploit the application characteristics.

\section{REFERENCES}

[1] A. Perrig, R. Szewczyk, V. Wen, D. Culler, and J.D. Tygar, G. Eason, "SPINS: Security Protocols for sensor Networks," Wireless Networks, Vol.8, pp.521-534, 2002, doi:10.1023/A:1016598314198

[2] R. Chandramouli, S. Bapatla, K.P. Subbalakshmi, and R.N. Uma, "Battery Power_Aware Encryption", ACM Transactions in Information and systems Security, Vol.9, No.2, pp.162-180, May 2006.

[3] B. Soewito, L. Vespa, and N. Weng, "Characterizing Power and Resource Consumption of Encryption/Decryption in Portable Devices," Region 5 IEEE Conf. April 2008, pp.1-6, doi:10.1109/TPSD.2008.4562749.

[4] M.A. Haleem, C.N. Mathur, R. Chandramouli, and K.P Subbalakshmi, "Opportunistic Encryption: A Trade-Off between Security and Throughput in Wireless Networks," IEEE Trans. On Dependable and Secure Computing, Vo.4, No.4, Oct-Dec 2007, pp.313-324.

[5] R. Hasimoto-Beltran, "High-performance multimedia encryption system based on chaos," Chaos 18, 023110, 2008.

[6] L. Kocarev, "Chaos-based Cryptography: a brief overview," IEEE Circuits Syst. Mag., Vol. No.6, 2001.

[7] PolarSSL, "http://polarssl.org".

[8] B. Gladman, "AES and combined encryption /authentication modes," 2006, http://fp.gladman.plus.com/AES/.

[9] D.J. Bernstein and P. Schwabe, "New AES Software Speed Records," INDOCRYPT-2008, LNCS Vol. 5365, 2008, pp. 322-336. 
[10] National Institute of Standards and Technology (NIST), “,FIPS-197: Advanced http://www/csrc.nist.gov/publications/fips/fips197/fips-197.pdf.
[11] D.A. Osvik, J.W. Bos, D. Stefan, and D. Canright, "Fast Software AES Encryption," 17th International Workshop on Fast Software Encryption, 2010.

[12] Maemo,http://maemo.org/

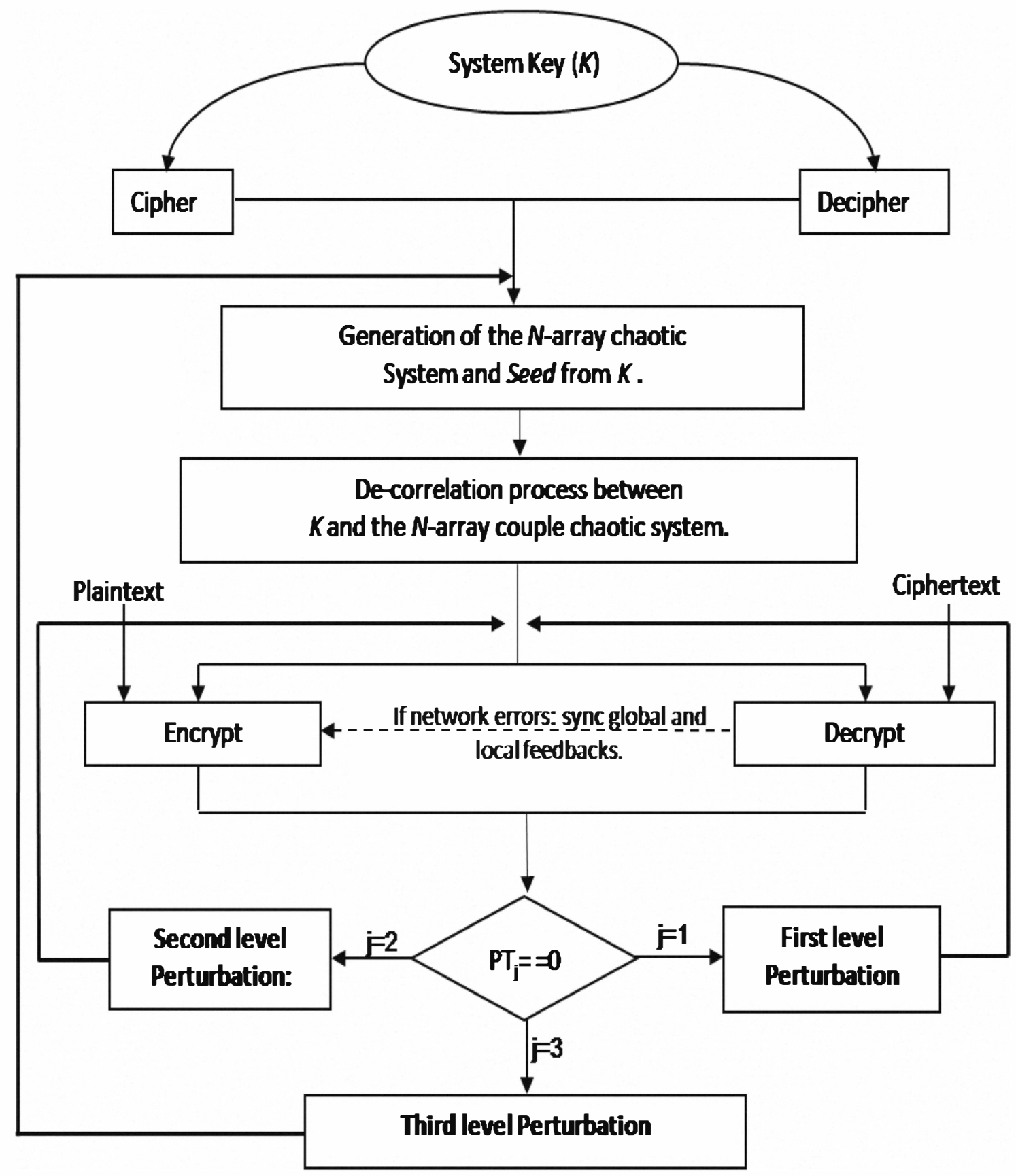

Figure 1: Flow diagram for the chaotic encryption/decryption system [5]. 

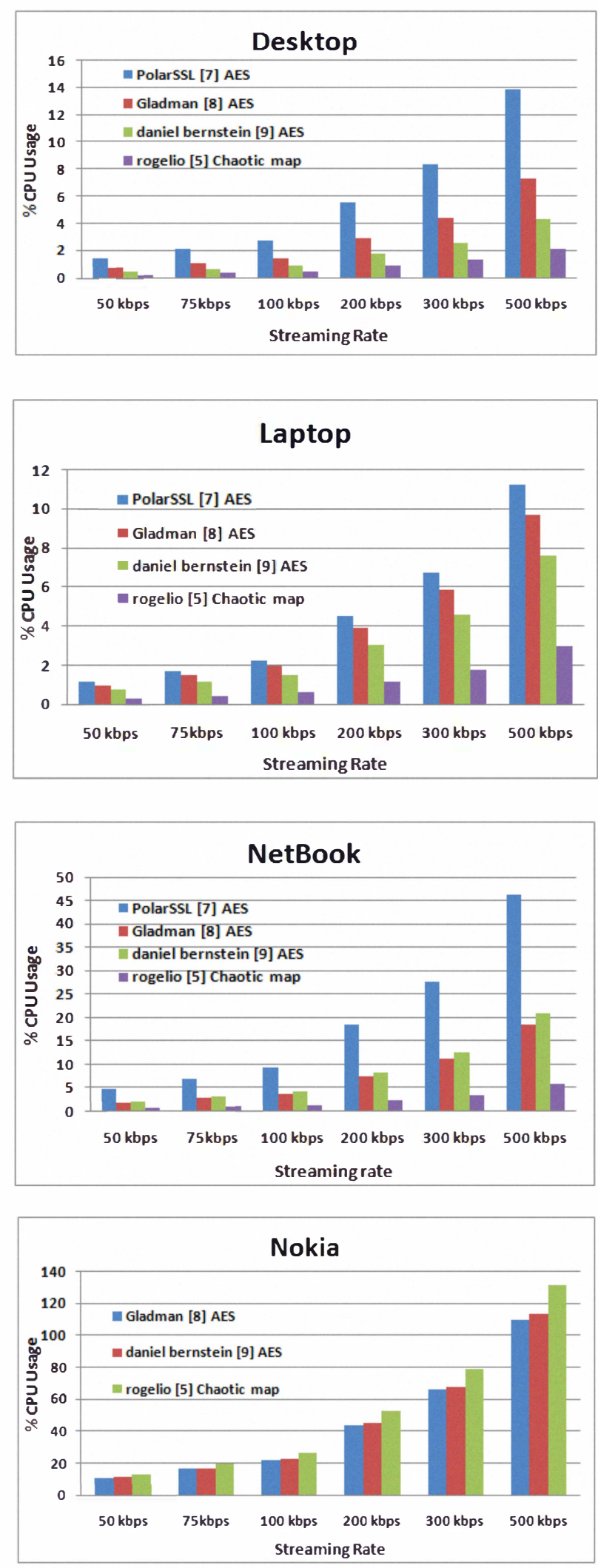

a) CPU Usagefor different streaming rates

Figure 2: Performance results on different platforms in terms of encryption speed and CPU usage.
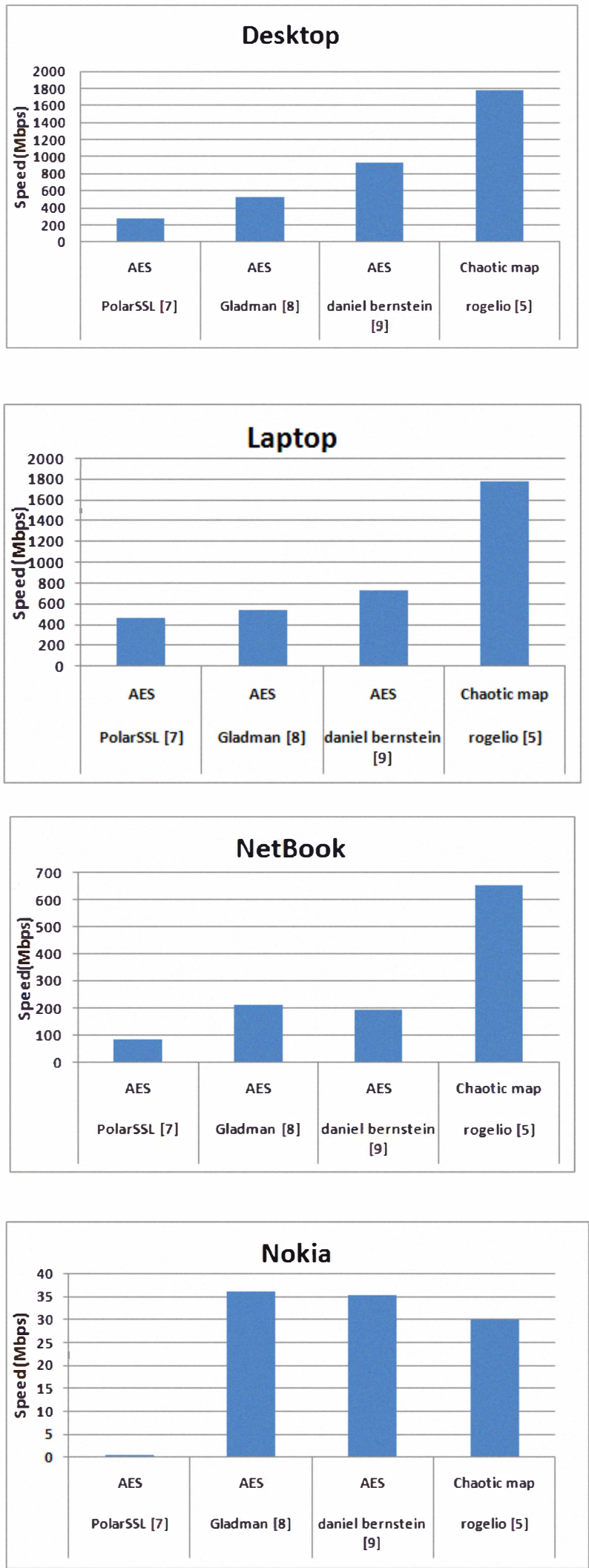

b) Encryption Speeds 


\section{LAPTOP}
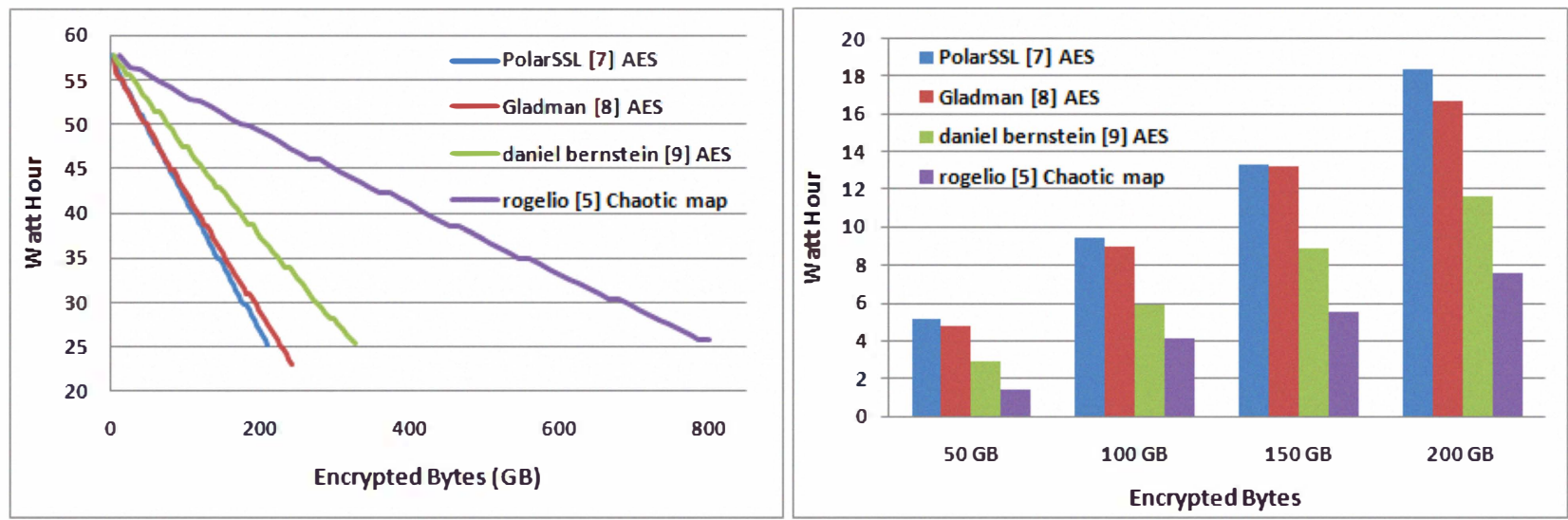

\section{Netbook}

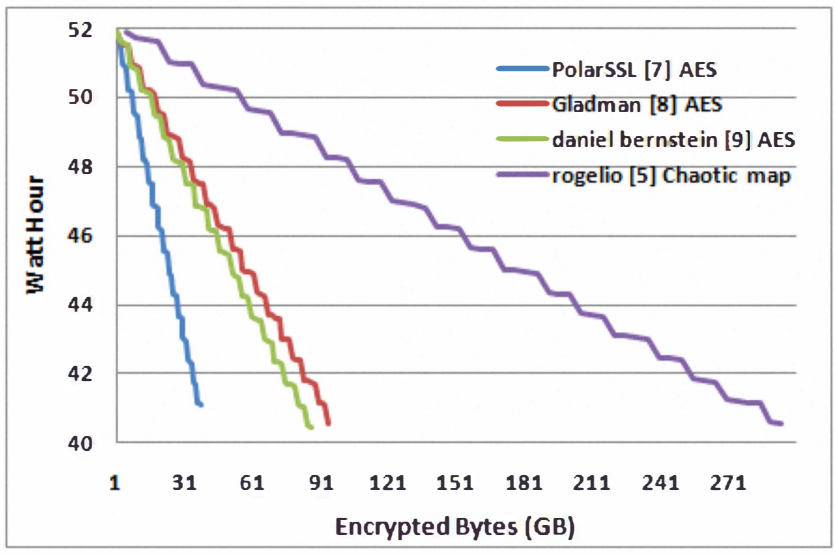

(a)

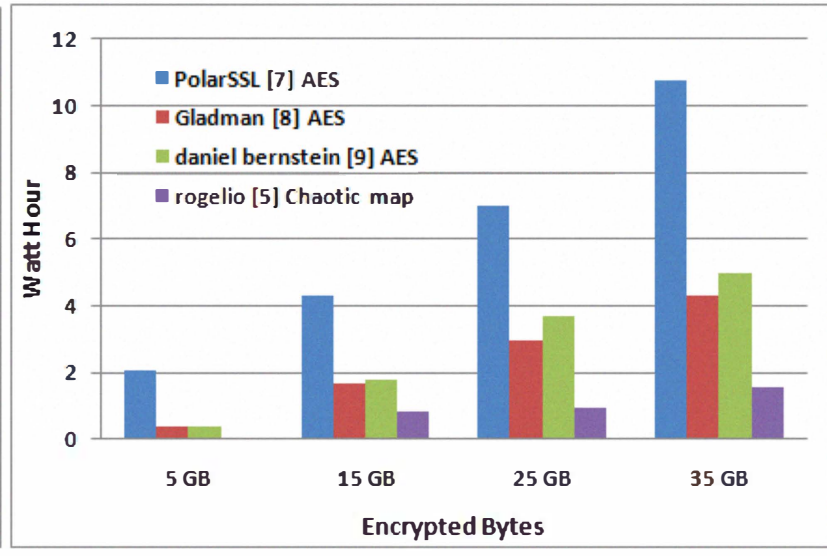

(b)

Figure 3: Performance results in terms of energy consumed for: a) encryption running for a fixed time period (60 minutes) and corresponding bytes encrypted, b) encrypting different data sizes. 